\title{
Plasma homocysteine, apolipoprotein E status and vascular disease in elderly patients with mental illness
}

\author{
Karin Nilsson ${ }^{1}$, Lars Gustafson¹, Michael Nornholm¹ \\ and Björn Hultberg ${ }^{2, *}$ \\ ${ }^{1}$ Department of Psychogeriatrics, Clinical Science, Lund \\ University Hospital, Lund, Sweden \\ ${ }^{2}$ Division of Clinical Chemistry, Laboratory Medicine, \\ Lund University Hospital, Lund, Sweden
}

\begin{abstract}
Background: Total plasma homocysteine (tHcy) concentration is increased in elderly patients with mental illness. Also, patients with vascular disease have significantly higher plasma tHcy concentration compared with patients without vascular disease. Apolipoprotein E (apoE) status is associated with cardiovascular disease and a major genetic risk factor is inheritance of the $e 4$ allele. In the present study, we investigated the association between plasma tHcy and apoE status. Methods: The relation between apoE status, plasma tHcy and vascular disease was investigated in a cohort of consecutively enrolled elderly patients with mental illness $(n=328)$. Results: Plasma tHcy concentrations were increased $(\mathrm{p}<0.01)$ in carriers of APOE4 $(13.6 \mu \mathrm{mol} / \mathrm{L} ; 9.2-21.7$ $\mu \mathrm{mol} / \mathrm{L})$ compared to non-carriers $(12.4 \mu \mathrm{mol} / \mathrm{L} ; 8.3-19.9$ $\mu \mathrm{mol} / \mathrm{L})$. The proportion of patients with vascular disease was significantly $(\mathrm{p}<0.001)$ increased among carriers $(61 \%)$ compared to non-carriers (42\%). An increased percentage $(\mathrm{p}<0.001)$ of APOE4 carriers was observed in patients with Alzheimer's disease (AD) with $(71 \%)$ or without vascular disease $(42 \%)$, and in patients with vascular dementia (VaD) (54\%) compared to a reference group (34\%).

Conclusions: Since carriers of APOE4 showed an increased likelihood of vascular disease, these patients need more intensive control of other modifiable vascular risk factors. Furthermore, the association between plasma tHcy and the presence of APOE4 might be attributed to an increased proportion of vascular disease in APOE4 carriers.

Clin Chem Lab Med 2010;48:129-35.
\end{abstract}

*Corresponding author: B. Hultberg, Department of Clinical Chemistry, Institute of Laboratory Medicine, University Hospital, S-22185 Lund, Sweden

Phone: +46 46173 447, Fax: +46 46189 114,

E-mail: bjorn.hultberg@med.lu.se

Received April 2, 2009; accepted September 15, 2009;

previously published online November 30, 2009
Keywords: apolipoprotein E; cobalamin; creatinine; folate; homocysteine; psychogeriatric patients; vascular disease.

\section{Introduction}

Apolipoprotein E (apoE) plays a central role in lipid metabolism as a component of very low-density lipoproteins and chylomicrons (1). The APOE gene, located at chromosome 19 , is polymorphic with three common alleles $-e 2, e 3$ and $e 4$ (2). One genetic risk factor associated with late-onset Alzheimer's disease (AD) is inheritance of the $e 4$ allele $(2,3)$. This allele is also associated with cardiovascular disease, unlike the other two common alleles $e 2$ and $e 3$ (2-4).

Many studies have reported significantly higher total plasma homocysteine (tHcy) in elderly patients with mental illness compared with control subjects (5-8). The concentration of plasma tHcy is influenced by several factors and their interaction $(9,10)$. Some of these factors are age, cobalamin/ folate status, renal function and the presence of vascular disease. Many clinical and epidemiological studies published during the last 20 years show that even mild hyperhomocysteinemia is associated with vascular disease (9, 11-16). Therefore, we studied elderly patients with mental illness with respect to the presence of vascular disease (5, 17-20). Briefly, our findings showed that patients with mental illness and vascular disease had significantly higher plasma tHcy concentrations compared with patients with mental illness but without vascular disease (5, 17-20), and that increased plasma tHcy was associated primarily with the presence of vascular disease and not related to a specific psychogeriatric diagnosis.

Vascular disease plays an important role in cognitive impairment in elderly patients with mental illness (21). There is a complex relation between vascular disease and dementia (22). Vascular dementia ( VaD) and AD together account for the vast majority of cases of dementia. $\mathrm{AD}$, the most common type of degenerative dementia, has long been considered to be a primarily degenerative brain disease. However, many patients with $\mathrm{AD}$ also have vascular disease contributing to brain pathology (23). An increasing number of epidemiological studies have provided further support for the suggestion that the presence of vascular disease or traditional vascular risk factors is associated with $\mathrm{AD}$ (24-27). VaD is considered to be the second most common cause of dementia and may result from several different types and locations of cerebrovascular lesions. The coexistence of $\mathrm{VaD}$ and $\mathrm{AD}$ may be the most common cause of dementia, i.e., mixed 
dementia where both degenerative and vascular injuries are present $(23,28)$.

The public health burden of $\mathrm{AD}$ and $\mathrm{VaD}$ threatens to soar in the next few decades. There is a growing need to modify known risk factors for cognitive dysfunction, and one modifiable risk factor is vascular disease $(21,22)$. Proper control of vascular disorders may prevent or delay the onset of cognitive impairment $(21,22,29)$. We have previously investigated different markers for vascular disease and their relation to the presence of vascular disease and plasma tHcy concentrations in consecutively enrolled elderly patients with mental illness (29-31). In the present study, we further extend those studies by investigating the relation between apoE status, a genetic risk marker of vascular disease, the presence of vascular disease and plasma tHcy concentrations in elderly patients with mental illness. By investigating markers for vascular disease in these patients, it might be possible to identify patients in need of more intensive control of their vascular disease and thereby prevent or delay cognitive impairment.

\section{Materials and methods}

\section{Study populations}

The present study population consisted of 328 patients consecutively enrolled (151 males and 177 females, 69 (51-83) years, median and 10th-90th percentiles) who were referred to the Psychogeriatric Department at Lund University Hospital for diagnostic assessments and treatment. Patients on any type of ongoing vitamin therapy were excluded from the study. The diagnosis was based on psychiatric, neurological, somatic, laboratory investigations, psychometric testing, measurements of regional cerebral blood flow, electroencephalography, and computer tomography scan (CT) or magnetic resonance imaging (5). The majority of cases were living in their own homes, alone or with relatives. The diagnosis of the psychogeriatric diseases was based on the DSM-IV criteria (32). Furthermore, patients with VaD fulfilled the NINDS-AIREN criteria (33) for $\mathrm{VaD}$. Patients with $\mathrm{AD}$ were diagnosed in accordance with the NINCDS-ADRDA criteria (34). Organic dementia was diagnosed in 146 patients, 61 patients were diagnosed with $\mathrm{AD}, 11$ patients with frontal lobe degeneration of non-Alzheimer type (FTD), 39 patients with $\mathrm{VaD}, 17$ patients with mixed vascular and Alzheimer type dementia (MX), 13 patients with other types of dementia (Oth Dem) and five patients with dementia NUD. One hundred and eighty-two patients suffered from non-organic mental diseases, two patients had delirium (Del), 23 patients depression (Depr), 13 patients other psychiatric disorders (Oth Psych, mainly psychosis), and 85 patients had mild cognitive impairment (MCI) (35). In addition, 59 patients presented with subjective symptoms of cognitive impairment but normal psychometric tests. They had normal CT and otherwise were apparently healthy. These patients were treated as a separate group of patients called subjective cognitive impairment (SCI). The APOE genotypes in the different diagnostic groups are shown in Table 1. The study was approved by the Ethical Committee of the University of Lund. Informed consent to participate was given by all subjects (or relatives if the patients were unable to communicate).

The patients were divided into two groups according to the presence or absence of vascular disease. Both cerebral and extra-cerebral vascular diseases were included in the group of patients with vascular disease. The presence of vascular disease in the patients was based on diagnosis and/or symptoms indicating vascular disease as indicated in their medical records. One group $(n=162)$ consisted of patients with vascular disease and included diagnoses/symptoms, such as $\mathrm{VaD}$, cerebral infarction, transitory ischaemic attacks, myocardial infarction, angina pectoris, peripheral vascular disease, atrial fibrillation, and hypertension. In another group of 166 patients, there was no indication of any vascular disease.

\section{Assays}

Blood samples for homocysteine determination were collected in evacuated tubes containing EDTA at about 8 a.m. following an

Table 1 Diagnoses of patients with and without vascular disease according to APOE status.

\begin{tabular}{|c|c|c|c|c|c|c|c|}
\hline & $\begin{array}{l}\text { Number of } \\
\text { patients }\end{array}$ & APOE $2 / 2$ & APOE $2 / 3$ & APOE $2 / 4$ & APOE $3 / 3$ & APOE3/4 & APOE $4 / 4$ \\
\hline Number of genotypes & 328 & 3 & 37 & 12 & 150 & 95 & 31 \\
\hline Dementia of Alzheimer type & 61 & & 5 & & 22 & 22 & 12 \\
\hline $\begin{array}{l}\text { Mixed vascular and Alzheimer } \\
\text { type dementia }\end{array}$ & 17 & & 2 & 2 & 4 & 7 & 2 \\
\hline $\begin{array}{l}\text { Frontal lobe degeneration of } \\
\text { non-Alzheimer type }\end{array}$ & 11 & & 2 & 1 & 4 & 2 & 2 \\
\hline Vascular dementia & 39 & & 3 & 4 & 15 & 15 & 2 \\
\hline Unspecified dementia & 5 & & & & 4 & & 1 \\
\hline $\begin{array}{l}\text { Other specified types of } \\
\text { dementia }\end{array}$ & 13 & & 1 & & 5 & 6 & 1 \\
\hline Delirium & 2 & & & & 2 & & \\
\hline Depression & 23 & 1 & 2 & 1 & 13 & 6 & \\
\hline $\begin{array}{l}\text { Other non-organic psychiatric } \\
\text { disorders }\end{array}$ & 13 & & 1 & & 9 & 1 & 2 \\
\hline Mild cognitive impairment & 85 & 1 & 11 & 3 & 44 & 21 & 5 \\
\hline Subjective cognitive impairment & 59 & 1 & 10 & 1 & 28 & 15 & 4 \\
\hline No vascular disease & 166 & 1 & 20 & 3 & 91 & 40 & 11 \\
\hline Vascular disease & 162 & 2 & 17 & 9 & 59 & 55 & 20 \\
\hline
\end{tabular}


overnight fast and centrifuged within $15 \mathrm{~min}$ at $3000 \times \mathrm{g}$ for $5 \mathrm{~min}$. Plasma tHcy was measured with HPLC after reduction of disulphide bonds with dithiothreitol and deproteinisation with sulphosalicylic acid (36). The upper reference limit (95th percentile) for plasma tHcy in a healthy elderly population without folate fortification is $19.9 \mu \mathrm{mol} / \mathrm{L}(17,18)$.

$A P O E$ genotyping was performed using a method of restriction genotyping as described by Hixson (37). The percentage of APOE4 carriers (APOE2/E4, E3/E4 and E4/E4) in a healthy Swedish population is about $30 \%$ (38).

Serum cobalamin (S-Cob) and serum folate (S-Fol) concentrations were determined using competitive protein binding assays (Roche Diagnostics, Mannheim, Germany; Modular Analytics E170). For S-Cob we used the reference interval of 150-650 $\mathrm{pmol} / \mathrm{L}$ (5). The reference interval according to the manufacturer (Roche Diagnostics) for S-Fol in individuals without folate fortification is 7-39 $\mathrm{nmol} / \mathrm{L}$.

Serum creatinine (S-Creat) concentrations (upper reference limit $105 \mu \mathrm{mol} / \mathrm{L}$ for males and $95 \mu \mathrm{mol} / \mathrm{L}$ for females) were measured with an enzymatic method (Roche Diagnostics; Modular Analytics P).

Serum C-reactive protein (CRP) (upper reference limit $3.0 \mathrm{mg} / \mathrm{L}$ ) was measured in a subpopulation of the present study population $(\mathrm{n}=58)$ with a routine method (Roche Diagnostics; Modular Analytics P).

\section{Statistics}

The results are presented as medians and 10th-90th percentiles. The following two-tailed tests at the 5\% level of significance were used to evaluate the study: Kruskal-Wallis one-way analysis of variance with Bonferoni-correction in cases with three or more independent samples, Mann-Whitney U-test for two independent samples. The $\chi^{2}$-test was used for comparison of frequencies between APOE4 carriers and non-carriers (Table 3 ) and percentage of APOE4 carriers in different diagnostic groups (Table 4). A general linear model (GLM) was used to identify factors with effects on plasma tHcy concentrations. Logistic regression analysis was performed in which the presence or absence of vascular disease was included as the dependent variable.

\section{Results}

The most frequent $A P O E$ genotype was $e 3 / e 3(\mathrm{n}=150)$, and e3/e4 $(\mathrm{n}=95)$, followed in descending order by e3/e2 $(\mathrm{n}=37), e 4 / e 4(\mathrm{n}=31), e 4 / e 2(\mathrm{n}=12)$, and $e 2 / e 2(\mathrm{n}=3)$. Diagnoses and patients with and without vascular disease in the different apoE status are shown in Table 1. Plasma tHcy concentrations were increased $(\mathrm{p}<0.001)$ in patients with vascular disease $(14.1 ; 9.1-21.2 \mu \mathrm{mol} / \mathrm{L}, \mathrm{n}=162)$ compared with patients without vascular disease (11.6; 8.5-19.3,
Table 3 Plasma tHcy, serum cobalamin (S-Cob), serum folate (SFol), serum creatinine (S-Creat), patients with and without vascular disease, and patients with dementia and non-dementia in APOE4 carriers and non-carriers.

\begin{tabular}{lll}
\hline & Non-carriers & Carriers \\
\hline Number & 190 & 138 \\
P-tHcy, $\mu \mathrm{mol} / \mathrm{L}$ & $12.4(8.3-9.9)$ & $13.6(9.2-21.7)^{\mathrm{b}}$ \\
S-Cob, pmol/L & $307(183-468)$ & $291(177-486)$ \\
S-Fol, nmol/L & $13.0(7.5-25.3)$ & $14.0(8.2-22)$ \\
S-Creat, $\mu$ mol/L & $75(58-108)$ & $78(57-114)$ \\
Vascular disease, $\mathrm{n}$ & $78(41 \%)$ & $84(61 \%)^{\mathrm{a}}$ \\
No vascular disease, $\mathrm{n}$ & $112(59 \%)$ & $54(39 \%)^{\mathrm{a}}$ \\
Dementia, $\mathrm{n}$ & $67(35 \%)$ & $79(57 \%)^{\mathrm{b}}$ \\
Non-dementia, n & $123(65 \%)$ & $59(43 \%)^{\mathrm{b}}$ \\
\hline
\end{tabular}

${ }^{\mathrm{a}} \mathrm{p}<0.001 ;{ }^{\mathrm{b}} \mathrm{p}<0.01$. Non-carriers are compared to carriers. Median, 10th-90th percentiles are presented for plasma tHcy, S-Cob, S-Fol and S-Creat, whereas number and percentage are presented for the patients.

$\mathrm{n}=166$ ). Plasma tHcy concentrations and age according to $A P O E$ status are shown in Table 2. There were no significant differences in these variables between the different $A P O E$ genotypes.

Table 3 shows a comparison of APOE4 carriers to noncarriers. Plasma tHcy concentrations were increased in carriers compared to non-carriers. Age correction of plasma tHcy did not change the significant difference. There was no significant difference between carriers and non-carriers with respect to use of drugs that may affect plasma tHcy concentrations such as folate, cobalamin or vitamin B6 antagonists. No association with APOE4 status was observed with respect to S-Creat, S-Cob or S-Fol. The proportion of patients with vascular disease was significantly increased among carriers compared to non-carriers, whereas patients without vascular disease exhibited a significantly increased proportion among non-carriers compared to carriers. Likewise, patients with dementia exhibited a significantly higher proportion among carriers compared to non-carriers, and patients without dementia exhibited a significantly increased proportion among non-carriers compared to carriers.

In Table 4, the percentage of APOE4 carriers is presented in some diagnostic groups and compared to patients with SCI, since these patients exhibited a normal percentage of APOE4 carriers. Patients with $\mathrm{AD}$, with or without vascular disease, showed a significantly increased percentage of APOE4 carriers compared to patients with SCI. Patients with $\mathrm{AD}$ and vascular disease also showed an increased percentage of APOE4 carriers $(\mathrm{p}>0.001)$ compared to AD patients

Table 2 Plasma tHcy concentration and age according to APOE status.

\begin{tabular}{lllllll}
\hline & APOE2/2 & APOE2/3 & APOE2/4 & APOE3/3 & APOE3/4 & APOE4/4 \\
\hline Number of genotypes & 3 & 37 & 12 & 150 & 95 & 31 \\
P-tHcy, $\mu$ mol/L & $13.5(5.8-16.2)$ & $12.8(8.8-20.1)$ & $12.9(8.5-24.1)$ & $12.4(8.3-20)$ & $13.9(9.3-22.5)$ & $13.1(9.1-20)$ \\
Age, years & $73(60-86)$ & $68(49-84)$ & $72(55-83)$ & $64(49-84)$ & $72(53-83)$ & $71(53-81)$ \\
\hline
\end{tabular}

Median, 10th-90th percentiles are presented. 
Table 4 Age, plasma tHcy, and percentage of APOE4 carriers in different diagnostic groups.

\begin{tabular}{|c|c|c|c|c|}
\hline & Number & Age, years & P-tHcy, $\mu \mathrm{mol} / \mathrm{L}$ & $\begin{array}{l}\text { APOE4 } \\
\text { carriers, \% }\end{array}$ \\
\hline $\mathrm{AD}$ & 61 & $72(54-87)^{\mathrm{a}}$ & $15.0(10.3-21.6)^{\mathrm{a}}$ & $56^{\mathrm{a}}$ \\
\hline $\mathrm{AD}$ with vascular disease & 28 & $72(61-84)^{\mathrm{a}}$ & $15.1(10.7-20.5)^{\mathrm{b}}$ & $71^{\mathrm{a}}$ \\
\hline AD without vascular disease & 33 & $71(52-90)^{\mathrm{a}}$ & $14.5(10-22.8)^{\mathrm{c}}$ & $42^{\mathrm{a}}$ \\
\hline MCI & 85 & $62(50-77)^{\mathrm{c}}$ & $11.7(8.8-17.3)^{\mathrm{c}}$ & 34 \\
\hline MCI with vascular disease & 40 & $69(54-78)^{\mathrm{a}}$ & $12.5(9-21.5)^{\mathrm{c}}$ & 37 \\
\hline MCI without vascular disease & 45 & $57(47-72)$ & $11.3(8.4-16.1)$ & 31 \\
\hline $\mathrm{VaD}$ & 39 & $80(69-87)^{\mathrm{a}}$ & $17.0(9.5-25.7)^{\mathrm{a}}$ & $54^{\mathrm{a}}$ \\
\hline Depr & 29 & $66(48-84)^{\mathrm{c}}$ & $11.4(7.4-16.7)$ & 30 \\
\hline SCI & 59 & $57(41-73)$ & $10.7(7.8-15.7)$ & 34 \\
\hline
\end{tabular}

${ }^{\mathrm{a}} \mathrm{p}<0.001 ;{ }^{\mathrm{b}} \mathrm{p}<0.01 ;{ }^{\mathrm{c}} \mathrm{p}<0.05$. The different diagnostic groups are compared with patients with SCI. Kruskal-Wallis and Mann-Whitney tests were used to detect significant changes in plasma tHcy levels and age. Median, 10th-90th percentiles are presented for age and plasma tHcy, whereas percentages are presented for APOE4 carriers.

without vascular disease. Patients with MCI, with or without vascular disease, exhibited no significant increase in carriers compared to patients with SCI, whereas patients with VaD showed an increased percentage of APOE4 carriers compared to patients with SCI.

Plasma tHcy concentrations were increased in AD patients with or without vascular disease, in MCI patients with vascular disease, and in patients with $\mathrm{VaD}$ compared to patients with SCI (Table 4). All diagnostic groups, except MCI without vascular disease, exhibited older age than patients with SCI. In addition, there were no significant differences between S-Cob, S-Fol or S-Creat among the different diagnostic groups shown in Table 4 (data not shown).

In a subgroup that included 58 randomly selected patients, 43 had normal serum CRP and 15 had increased serum CRP (above $3 \mathrm{mg} / \mathrm{L}$ ). Carriers of APOE4 with both normal and increased serum CRP showed no significant changes in plasma tHcy concentrations compared to non-carriers (normal CRP: carriers $12.5,7.9-23.7 \mu \mathrm{mol} / \mathrm{L}, \mathrm{n}=20$ and non-carriers 10.4, 7.1-22.7, $\mathrm{n}=23$; increased CRP: carriers 13.3, 6.3-16.7 $\mu \mathrm{mol} / \mathrm{L}, \mathrm{n}=8$ and non-carriers 9.0, 6.7-16.0, $\mathrm{n}=8)$.

Multivariate analysis (GLM) that included age, S-Fol, SCob, S-Creat, and APOE4 status showed that age, S-Fol, SCreat and S-Cob significantly predicted plasma tHcy concentrations (Table 5). Logistic regression analysis in all patients, with the presence or absence of vascular disease as the dependent variable, showed that plasma tHcy, adjusted for its significant predictors (age, S-Fol, S-Creat and S-Cob), and the presence of APOE4 status were significant predictors

Table 5 General linear model estimates of factors with potential impact on plasma tHcy concentrations (dependent variable).

\begin{tabular}{lrr}
\hline Independent variable & t-Value & $\mathrm{p}$-Value \\
\hline Age & 5.588 & $<0.001$ \\
S-Fol & -5.293 & $<0.001$ \\
S-Cob & -2.079 & 0.039 \\
S-Creat & 6.259 & $<0.001$ \\
Presence of APOE4 status (yes/no) & -0.193 & 0.847 \\
\hline
\end{tabular}

of vascular disease (adjusted plasma tHcy - t-value 3.413, $\mathrm{p}<0.001$; presence of APOE4 status - t-value 2.959, $\mathrm{p}<0.01)$.

\section{Discussion}

The present study population consisted of consecutively enrolled patients with mental illness. Approximately half of the patients had co-existent vascular disease and $\sim 40 \%$ had dementia. The current study showed that in these elderly patients with mental illness, plasma tHcy concentrations were increased in $A P O E 4$ carriers compared to non-carriers. In previous investigations (39-41), no association was observed between plasma tHcy and APOE4 status. Those investigations, however, did not focus specifically on the association of interest, and two $(39,40)$ had small population sizes. In a recent study (42), APOE4 carriers in an elderly healthy population had an even lower risk of hyperhomocysteinemia than did non-carriers, but only if the carriers did not have increased CRP concentrations. The authors (42) suggested that this finding could possibly be attributed to CRP and plasma tHcy competing for the same mechanism (perhaps a lipoprotein carrier) that favours their clearance from blood in APOE4 carriers. However, in a small subpopulation of the present study population we could observe no such association between APOE4 carrier status and concentrations of plasma tHcy and serum CRP. Carriers of APOE4 with both normal and increased serum CRP showed a tendency towards increased plasma tHcy (not significant) compared to non-carriers. The discrepant findings might possibly be explained by different selection criteria and different populations. In the present study, patients with vascular disease showed an increased proportion among APOE4 carriers, which is in agreement with previous studies (2-4). The increased proportion of patients with vascular disease in APOE4 carriers could explain the increased plasma tHcy concentrations in carriers, since the presence of vascular disease is associated with increased concentrations of plasma tHcy $(9,11-16)$. 
Clarification of the role of vascular risk factors for cognitive dysfunction is important since these may serve as targets for strategies for prevention $(21,22)$. Few studies have examined the effect of vascular risk factors on dementia progression after establishing a diagnosis of AD. Mielke et al. (43) have shown that atrial fibrillation, hypertension and angina pectoris were associated with a greater rate of decline and thus may represent modifiable risk factors for secondary prevention in AD. Likewise, Song et al. (44) observed that $\mathrm{AD}$ with silent cerebral infarction showed a more severe cognitive decline than $\mathrm{AD}$ without vascular disease, indicating that cerebrovascular disease contributes to the severity of cognitive decline. Also, Sheng et al. (45) showed that AD with coexisting cerebral infarction (satisfying criteria for $\mathrm{VaD})$ was associated with faster progression of dementia. These findings suggest that prevention of cerebrovascular disease may play an important role in preventing the rapid cognitive decline of $\mathrm{AD}$.

The percentage of APOE4 carriers in normal subjects has been reported to be about $30 \%$ (38). An increased percentage of APOE4 carriers was observed in $\mathrm{AD}$ patients with or without vascular disease, which is in agreement with previous studies $(2,3)$. In addition, AD patients with vascular disease exhibited a higher percentage (71\%) of APOE4 carriers than AD patients without vascular disease $(42 \%)$. In previous investigations, plasma tHcy concentrations were also observed to be increased in AD patients with vascular disease compared to AD patients without vascular disease (20, 29). ApoE4 might contribute to AD pathology by interacting with multiple factors through various pathways (46). For example, interactions with the amyloid $\beta$-peptide and the amyloid cascade may lead to cognitive decline and neurodegeneration. However, since patients with AD and vascular disease showed a higher percentage of APOE4 carriers than patients with $\mathrm{AD}$ without vascular disease, it is possible that apoE4 primarily reflects the presence of vascular disease. In agreement with the findings of Religa et al. (39), patients with MCI did not show an increased percentage of APOE4 carriers. Most previous studies have also demonstrated an association between APOE4 carriers and patients with $\mathrm{VaD}$ (47). Although not all studies found a significant association, some studies (47) that did not find a significant association did find a tendency towards a greater frequency of APOE4 carriers in $\mathrm{VaD}$. In the present study, we observed a significant increase in carriers. However, the number of patients with $\mathrm{VaD}$ was relatively small.

APOE4 carriers have been associated with cognitive decline in elderly subjects $(41,48)$. In accordance with these findings, we observed an increased frequency of patients with dementia in the group of APOE4 carriers compared to non-carriers. One possible reason for the association of the APOE4 genotype and cognitive impairment is that the APOE4 genotype is related to vascular disease which, in turn, is related to cognitive impairment (21).

In the present study, we have investigated APOE4 status as a possible risk marker for vascular disease in elderly patients with mental illness. APOE4 carriers showed an increased proportion in patients with vascular disease. Likewise, the presence of $A P O E 4$ status predicted the presence of vascular disease. The presence of APOE4 status in patients with mental illness might therefore be useful to identify patients at risk of rapid progression of vascular disease and consequently also of cognitive decline. Therefore, carriers of APOE4 might require more intensive control of other modifiable vascular risk factors, such as blood pressure or cholesterol. Furthermore, the association between plasma tHcy and the presence of apoE4 might be attributed to an increased proportion of vascular disease in APOE4 carriers.

\section{Conflict of interest statement}

Authors' conflict of interest disclosure: The authors stated that there are no conflicts of interest regarding the publication of this article. Research funding played no role in the study design; in the collection, analysis, and interpretation of data; in the writing of the report; or in the decision to submit the report for publication.

Research funding: This work was supported by grants from the Swedish Medical Research Council (grant no. 3950), the Alzheimer Foundation Sweden, the Sjöbring Foundation, the Swedish Heart Lung Foundation, the Albert Påhlsson Foundation and the County Council of Malmöhus.

Employment or leadership: None declared.

Honorarium: None declared.

\section{References}

1. Mahley RW, Rall SC. Apolipoprotein E. Far more than a lipid transport protein. Annu Rev Genom Hum G 2000;1:507-37.

2. Eichner JE, Dunn ST, Perveen GP, Thompson DM, Stewart KE, Stroehla BC. Apolipoprotein E polymorphism and cardiovascular disease: a huge review. Am J Epidemiol 2002;155:48795.

3. Poirer J. Apolipoprotein E, cholesterol transport and synthesis in sporadic Alzheimer's disease. Neurobiol Aging 2005;26:35561.

4. Davignon J, Cohn JS, Mabile L, Bernier L. Apolipoprotein E and atherosclerosis; insight from animal and human studies. Clin Chim Acta 1999;286:115-43.

5. Nilsson K, Gustafson L, Fäldt R, Andersson A, Brattström L, Lindgren A, et al. Hyperhomocysteinemia - a common finding in a psychogeriatric population. Eur J Clin Invest 1996;26: $853-9$.

6. Clarke R, Smith AD, Jobst KA, Refsum H, Sutton L, Ueland PM. Folate, vitamin B12, and serum total homocysteine levels in confirmed Alzheimer disease. Arch Neurol 1998;55:144955.

7. Lehmann M, Gottfries CG, Regland B. Identification of cognitive impairment in the elderly: homocysteine is an early marker. Dement Geriatr Cogn 1999;10:12-20.

8. Quadri P, Fragiacomo C, Pezzati R, Zanda E, Forloni G, Tettamanti $\mathrm{M}$, et al. Homocysteine, folate and vitamin B-12 in mild cognitive impairment, Alzheimer's disease and vascular dementia. Am J Clin Nutr 2004;80:114-22.

9. Refsum H, Smith DA, Ueland PM, Nexö E, Clarke R, McPartlin $\mathrm{J}$, et al. Facts and recommendations about total homocysteine 
determinations: an expert opinion. Clin Chem 2004;50:332.

10. Brattström L, Lindgren A, Israelsson B, Andersson A, Hultberg B. Homocysteine and cysteine: determinants of plasma levels in middle-aged and elderly subjects. J Intern Med 1994;236: 633-41.

11. Wald DS, Law M, Morris JK. Homocysteine and cardiovascular disease: evidence on causality from a meta-analysis. $\mathrm{Br}$ Med J 2002:325;1202-9.

12. Homocysteine Studies Collaboration. Homocysteine and risk of ischemic heart disease and stroke: a metaanalysis. J Am Med Assoc 2002;288:2015-22.

13. Wald DS, Morris JK, Law M, Wald NJ. Folic acid, homocysteine and cardiovascular disease: judging causality in the face of inconclusive trial evidence. Br Med J 2006;333:1114-7.

14. Herrmann W. Homocysteine research: alive and kicking! Clin Chem Lab Med 2007;45:1571-4.

15. Ueland PM, Clarke R. Homocysteine and cardiovascular risk: considering the evidence in the context of study design, folate fortification, and statistical power. Clin Chem 2007;53:807-9.

16. Castro R, Rivera I, Blom HJ, Jakobs C, Tavares de Almeida I. Homocysteine metabolism, hyperhomocysteinaemia and vascular disease: an overview. J Inher Metab Dis 2006;29:3-20.

17. Nilsson K, Gustafson L, Hultberg B. Plasma homocysteine concentration and its relation to symptoms of vascular disease in psychogeriatric patients. Dement Geriatr Cogn 2005;20:5-41.

18. Nilsson K, Gustafson L, Hultberg B. Plasma homocysteine and vascular disease in elderly patients with psychogeriatric disease. Dement Geriatr Cogn 2006;21:148-54.

19. Nilsson K, Gustafson L, Hultberg B. Plasma homocysteine, cobalamin/folate status, and vascular disease in a large population of psychogeriatric patients. Dement Geriatr Cogn 2006: 22:358-66.

20. Nilsson K, Gustafson L, Hultberg B. Elevated plasma homocysteine concentration in elderly patients with mental illness is mainly related to the presence of vascular disease and not the diagnosis. Dement Geriatr Cogn 2007;24:162-8.

21. Alagiakrishnan A, McCracen P, Feldman H. Treating vascular risk factors and maintaining vascular health: is this the way towards successful cognitive ageing and preventing cognitive decline? Postgrad Med J 2006;82:101-5.

22. Mielke MM, Zandi PP. Hematologic risk factors of vascular disease and their relation to dementia. Dement Geriatr Cogn 2006;21:335-52.

23. Brun A. The neuropathology of vascular dementia and its overlap with Alzheimer's disease. In: O'Brien J, Ames D, Gustafson L, Folstein M, Chiu E, editors. Cerebrovascular disease, cognitive impairment and dementia, 2nd ed. Martin Dunitz: Taylor \& Francis Group, 2004:103-15.

24. Skoog I, Lernfelt B, Landahl S, Palmertz B, Andreasson LA, Nilsson L, et al. 15-year longitudinal study of blood pressure and dementia. Lancet 1996;347:1141-5.

25. Hofman A, Ott A, Breteler MM, Bots ML, Slooter AJ, van Harskamp F, et al. Atherosclerosis apolipoprotein E, and prevalence of dementia and Alzheimers disease in the Rotterdam Study. Lancet 1997;349:151-4.

26. Ott A, Stolk RP, van Harskamp F, Pols HA, Hofman A, Breteler MM. Diabetes mellitus and risk of dementia: the Rotterdam Study. Neurology 1999;53:1937-42.

27. Rosendorff C, Beeri MS, Silverman JM. Cardiovascular risk factors for Alzheimer's disease. Am J Geriatr Cardiol 2007;16: $143-9$.
28. Korczyn AD. The underdiagnosis of the vascular contribution to dementia. J Neurol Sci 2005;229-230:3-6.

29. Nilsson K, Gustafson L, Hultberg B. Plasma homocysteine and vascular disease in elderly patients with mental illness. Clin Chem Lab Med 2008;46:1556-61.

30. Nilsson K, Gustafson L, Hultberg B. Homocysteine, cystatin C and $\mathrm{N}$-terminal-pro brain natriuretic peptide. Vascular risk markers in elderly patients with mental illness. Dement Geriatr Cogn 2007;25:88-96.

31. Nilsson K, Gustafson L, Hultberg B. C-reactive protein - vascular risk marker in elderly patients with mental illness. Dement Geriatr Cogn 2008;26:251-6.

32. American Psychiatric Association. DSM IV. Diagnostic and statistical manual of mental disorders, 4th ed. Washington, DC: American Psychiatric Association, 1994.

33. Roman GC, Tatemichi TK, Erkinjuntti T, Cummings JL, Masdeu JC. Vascular dementia: diagnostic criteria for research studies. Report of the NINDS-AIREN International workshop. Neurology 1993;43:250-60.

34. McKhann G, Drachman D, Folstein M, Katzman R, Price D, Stadlan M. Clinical diagnosis of Alzheimer's disease: report of the NINCDS-ADRDA Work Group under the auspices of Department of Health and Human Services Task Force on Alzheimer's Disease. Neurology 1984;34:939-44.

35. Petersen RC, Smith GE, Waring SC, Ivnik RJ, Tangalos EG, Kokmen E. Mild cognitive impairment: clinical characterization and outcome. Arch Neurol 1999;56:303-8.

36. Andersson A, Isaksson A, Brattström L, Hultberg B. Homocysteine and other thiols determined in plasma by HPLC and thiol-specific post-column derivatization. Clin Chem 1993; 39:1590-7.

37. Hixson JE. Apolipoprotein E polymorphisms affect atherosclerosis in young males. Arterioscler Thromb 1991;11:1237-44.

38. Eggertsen G, Tegelman R, Ericsson S, Angelin B, Berglumd L. Apolipoprotein E polymorphism in a healthy Swedish population: variation of allele frequency with age and relation to serum lipid concentrations. Clin Chem 1993;39:2125-9.

39. Religa D, Styczynska M, Peplonska B, Gabryelewicz T, Pfeffer A, Chodakowaska M, et al. Homocysteine, apolipoprotein E and methyleneterahydrofolate reductase in Alzheimer's disease and mild cognitive impairment. Dement Geriatr Cogn 2003; 16:64-70.

40. Lin SK, Kao JT, Tsai SM. Association of apolipoprotein E genotypes with serum lipid profiles in a healthy population of Taiwan. Am Clin Lab Sci 2004;34:443-8.

41. Elias MF, Robbins MA, Budge MM, Elias PK, Dore GA, Brennan SL, et al. Homocysteine and cognitive performance: modification by the ApoE genotype. Neurosci Lett 2008;430:4-69.

42. Ravaglia G, Forti P, Maioli F, Chiappelli M, Montesi F, Bianchin M, et al. Apolipoprotein E e4 allele affects risk of hyperhomocysteinemia in the elderly. Am J Clin Nutr 2006;84: 1473-80.

43. Mielke MM, Rosenberg PB, Tschanz J, Cook L, Hayden KM, Norton $\mathrm{M}$, et al. Vascular factors predict rate of progression in Alzheimer disease. Neurology 2007;69:1850-8.

44. Song IU, Kim JS, Eah KY, Lee KS. Clinical significance of silent cerebral infarctions in patients with Alzheimer disease. Cogn Behav Neurol 2007;20:93-8.

45. Sheng B, Cheng LF, Law CB, Li HL, Yeung KM, Lau KK. Coexisting cerebral infarction in Alzheimer's disease is associated with fast dementia progression: applying the National Institute for Neurological Disorders and Stroke/Association 
Internationale pour la Recherche et l'Enseignement en Neurosciences Neuroimaging Criteria in Alzheimer's Disease with Concomitant Cerebral Infarction. J Am Geriatr Soc 2007;55: 918-22.

46. Mahley RW, Huang Y, Weisgraber KH. Detrimental effects of apolipoprotein E4: potential therapeutic targets in Alzheimer's disease. Curr Alzheimer Res 2007;4:537-40.
47. Baum L, Lam LC, Kwok T, Lee J, Chiu HF, Mok VC, et al. Apolipoprotein E 4 allele is associated with vascular dementia. Dement Geriatr Cogn 2006;22:301-5.

48. Bretsky P, Guralnik L, Launer L, Albert M, Seeman TE. The role of ApoE-epsilon 4 in longitudinal cognitive decline: MacArthur studies of successful aging. Neurology 2003;60:107781. 